\title{
Massive Stars in the Galactic Center
}

\author{
F. Martins ${ }^{1} \dagger$, D. J. Hillier ${ }^{2}$, R. Genzel ${ }^{1,3}$, F. Eisenhauer ${ }^{1}$, T. Ott ${ }^{1}$, \\ S. Gillessen ${ }^{1}$ and S. Trippe ${ }^{1}$ \\ ${ }^{1} \mathrm{MPE}$, \\ Postfach 1312, D-85741, Garching, Germany \\ email: martins@graal .univ-montp2.fr \\ ${ }^{2}$ Dept. of Physics and Astronomy, University of Pittsburgh, \\ PA-15260 Pittsburgh, USA \\ ${ }^{3}$ Dept. of Physics, University of California, \\ CA-94720 Berkeley, USA
}

\begin{abstract}
We present results of two studies aiming at better understanding the properties of massive stars in the Galactic Center. We focus on the youngest and oldest of the three massive clusters harboring this region, namely the Arches and central cluster. We show that the development of powerful observational techniques in the near infrared spectral range (mainly 3D spectroscopy) allows to uncover the entire massive star population in these clusters. Using CMFGEN models, we derive the classical stellar and wind properties of 46 stars, as well as their surface abundances. The latter allow us to investigate in detail their evolutionary status and to identify evolutionary sequences between different types of stars. We thus constrain stellar evolution in the upper part of the HR diagram.
\end{abstract}

Keywords. stars: early-type - stars: Wolf-Rayet - stars: fundamental parameters - Galaxy: center

\section{Introduction}

The Galactic Center hosts three massive clusters: the Quintuplet, the Arches and the central cluster. They are all young $(2<$ age $<8 \mathrm{Myr})$, massive $\left(M>10^{4} \mathrm{M}_{\odot}\right)$ and consequently host a large number of massive stars: several hundreds of O stars, as well as about one third of the total number of Wolf-Rayet stars known in the Galaxy. A very interesting feature of the clusters is that they have different ages: the Arches cluster is the youngest (2-3 Myr), followed by the Quintuplet cluster (4 Myr) and the central cluster $(6 \mathrm{Myr})$. Hence, the contain different populations of massive stars and represent a unique opportunity to study massive star evolution. Here, we report on the detailed analysis of the massive star content of the youngest (Arches) and the oldest (central) cluster and provide improved evolutionary sequences for massive stars.

\section{The central cluster}

The central parsec of the Galaxy is a fascinating environment hosting the radio source and supermassive black hole Sgr A*. Its stellar content was first uncovered in the late eighties by Forrest et al. (1987) and Allen et al. (1990). They revealed the presence of bright infrared sources with strong emission lines in the K-band, especially the HeI 2.058 $\mu m$ line. At that time, such objects were observed only in the central parsec and they were thought to be peculiar to this region. This population of about 10 stars was subsequently

$\dagger$ Present address: GRAAL - CNRS - Université Montpellier II, Place Eugène Bataillon, 34095 Montpellier, France 


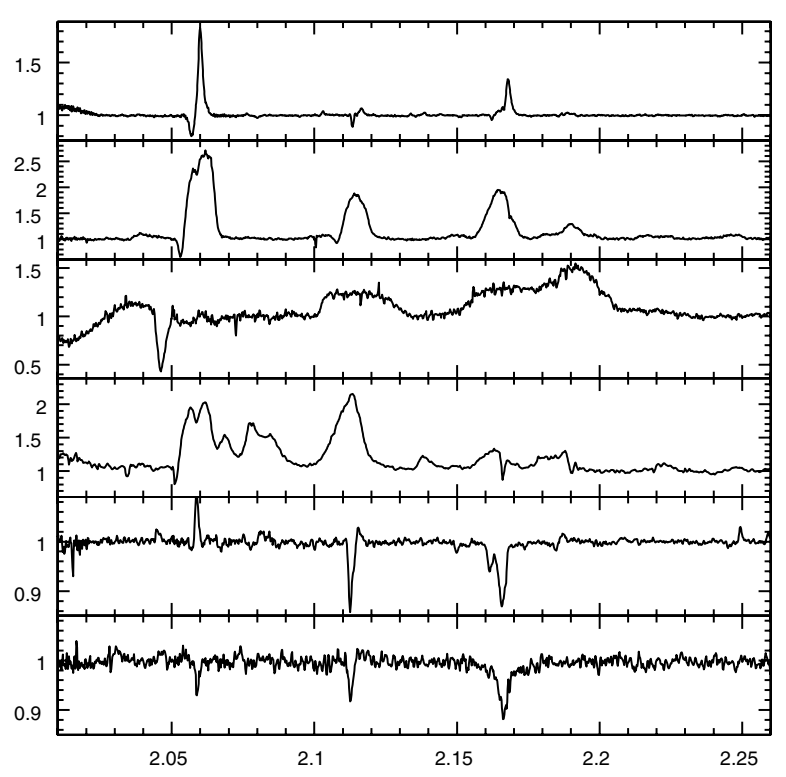

Figure 1. Mosaic of massive stars spectra observed in the central parsec with SINFONI. From top to bottom: IRS16C (Ofpe/WN9), IRS9W (WN8), IRS16SE2 (WN5-6), IRS7SE (WC9), average of the brightest $\mathrm{O}$ supergiants, average of $\mathrm{O}$ dwarfs.

analyzed by Najarro et al. (1994) and Najarro et al. (1997). They showed that the HeI emission line stars were luminous but rather cool $\left(T_{\text {eff }} \sim 25,000 \mathrm{~K}\right)$ massive stars in a post-main sequence evolutionary state. This population was somewhat puzzling since it was not producing enough ionizing photons to account for the nebular HeI emission. Besides, it was inconsistent with a 6-7 Myr starburst model, an age inferred from the very presence of these stars. Indeed, such a model produced many Wolf-Rayet stars not observed in the central cluster. This lead people to speculate that stellar evolution (the building block of starburst models) was failing to reproduce the central cluster population and that stellar evolution was proceeding differently in the Galactic Center. The alternative to this explanation was that the predicted population of hotter massive stars was not yet observed because it was fainter.

The solution to this puzzle came in the last few years. It benefited from two major improvements: new observational techniques/instruments and improved atmosphere models for hot stars. In terms of observational capabilities, the advent of adaptive optics assisted integral field spectrographs such as SINFONI on the ESO/VLT has allowed a detailed and deep (complete down to $\mathrm{mK} \sim 14$ ) spectroscopic mapping of the central parsec of the Galaxy. It resulted in the discovery of more than 100 new massive stars (a sample is shown in Fig. 1; see also Paumard et al. (2006)). Among them, one finds evolved late type $\mathrm{O}$ and early B supergiants, late type WC stars (mainly WC9), WN stars (WN5 to WN9) and even main sequence B stars such as the famous "S stars" (Ghez et al. (2003), Eisenhauer et al. (2005)). All these new stars are hotter than the HeI emission line stars. Consequently, there is a population of hot evolved stars as expected from a 7 Myr old starburst. Qualitatively, this tells us that there is probably no special evolution in this region. 


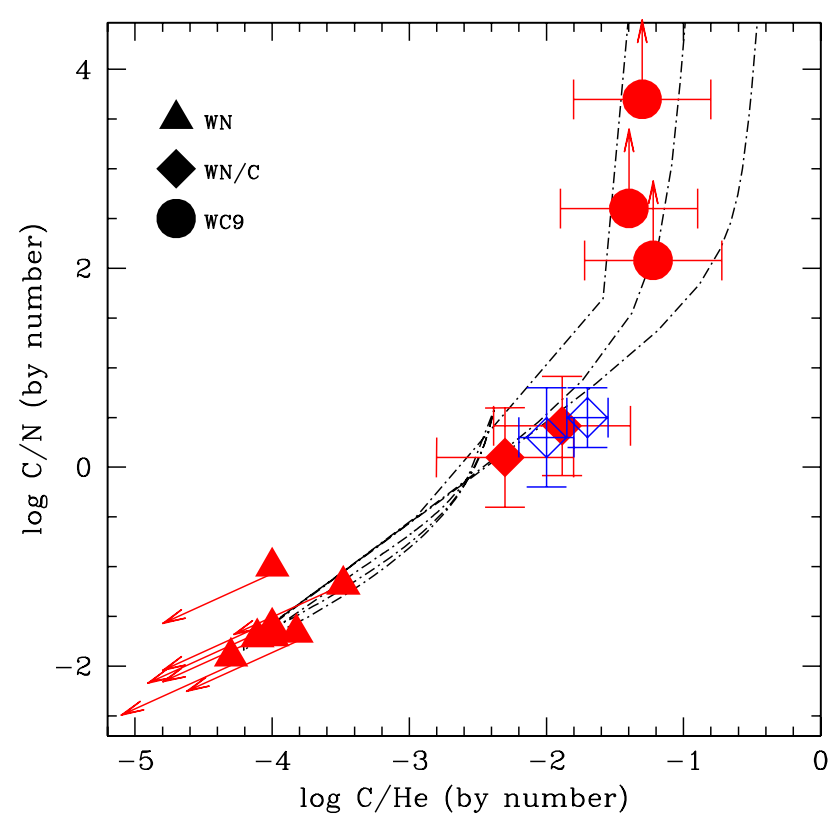

Figure 2. $\mathrm{C} / \mathrm{He}$ as a function of N/He for WN8 (triangles), WN8/WC9 (diamonds) and WC9 stars (circles). The Geneva evolutionary tracks with rotation are shown by the dot-dashed lines. The agreement between model predictions and derived properties is very good, indicating a normal stellar evolution in the central cluster.

To test this idea quantitatively, we used new generation atmosphere models to derive the stellar and wind properties of this new population (Martins et al. (2007)). The models were computed with the code CMFGEN (Hillier \& Miller(1998)) which includes a nonLTE treatment, spherical extension and winds, as well as line-blanketing. From the fit of observed K-band spectra, we derived the classical parameters: effective temperature, luminosity, mass loss rate, wind terminal velocity, ionizing flux. After summing up the contribution of all stars to obtain the total ionizing budget (using the calibrations and spectra of Martins et al. (2005) for stars of known spectral type but not analyzed directly in this study), we computed a photoionization model of the region with CLOUDY. We showed that the population of massive stars is now fully able to account for the nebular properties of the region. This is an indirect proof that this population is not peculiar, and that stellar evolution is most likely "normal".

A more direct evidence for a normal evolution came from the determination and analysis of surface abundances in post-main sequence. Using the amount of $\mathrm{H}, \mathrm{He}, \mathrm{C}$ and $\mathrm{N}$ observed at the surface of those stars, we were able to derive convincing evolutionary sequences between various types of stars. Fig. 2 illustrates this point. We show the $\mathrm{C} / \mathrm{He}$ ratio as a function of the $\mathrm{N} / \mathrm{He}$ ratio for $\mathrm{WN} 8$, transition $\mathrm{WN} 8 / \mathrm{WC} 9$ and $\mathrm{WC} 9$ stars. The carbon enrichment and nitrogen depletion from WN8 to WC9 stars is obvious. Most importantly, the Geneva evolutionary tracks including rotation shown by dot-dashed lines reproduce perfectly the observed sequence, confirming that standard stellar evolution accounts for the Galactic Center massive star population. 


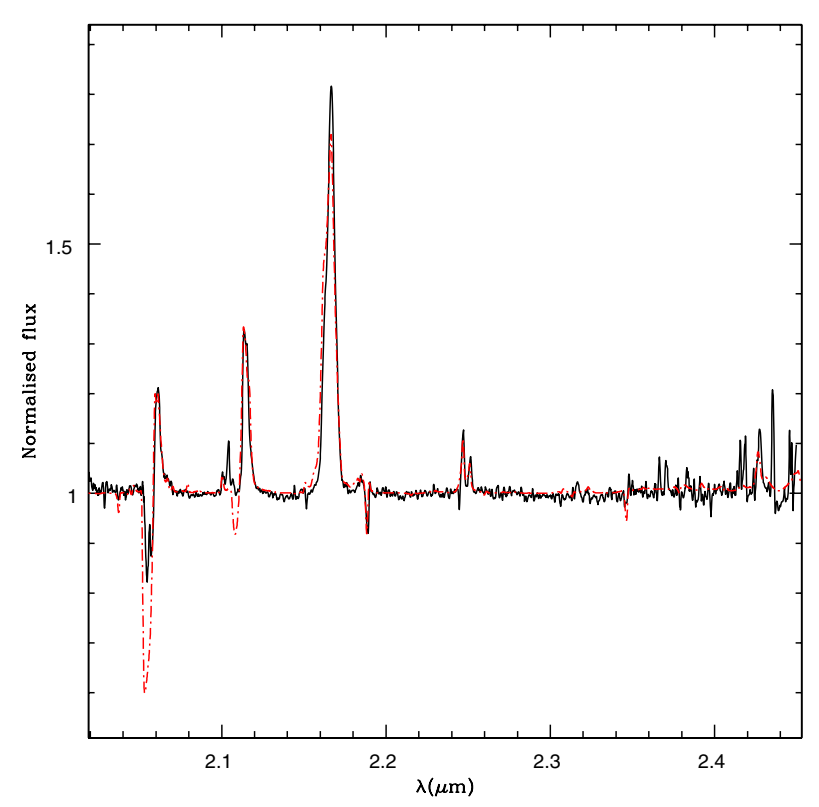

Figure 3. Best fit model (red dashed line) of the observed spectrum (black solid line) of a WN8-9h star in the Arches cluster.

\section{The Arches cluster}

The Arches cluster is the youngest of the three massive clusters in the Galactic Center (2-3 Myr, see Blum et al. (2001), Figer et al. (2002)). As such, it contains a different population of massive stars: early O stars and WN stars. Since they are rather young, they also have larger initial masses. Hence, studying the properties of these objects can bring new constraints on stellar evolution at very high luminosity and mass.

We have thus obtained SINFONI observations of the central part of the cluster as well as of bright stars located at larger distance. We have confirmed the presence of a large number of O4-6 supergiants, and we have improved the classification of the WN stars: they all appear to be WN7-9h stars, i.e. late type WN stars still showing a significant amount of hydrogen in their atmosphere (Martins et al. 2008).

As for the central cluster, we have derived the stellar and wind properties of the 28 brightest stars (Martins et al. 2008). A typical fit is shown in Fig. 3. They turned out to be all luminous objects, some of them reaching $10^{6.3} \mathrm{~L}_{\odot}$. In particular, all the WNLh stars are very luminous stars. Translated into initial masses, this means that they have progenitors with initial masses larger then $70 \mathrm{M}_{\odot}$, up to $120 \mathrm{M}_{\odot}$. These interesting objects are barely evolved in terms of He enrichment: some of them have the initial composition and are similar to O supergiants (see Fig. 5). However, all the WNLh stars show nitrogen enrichment and carbon depletion compared to O supergiants (see Martins et al. 2008). This is a strong indication that they are in an early state of evolution, already showing products of the CNO cycle at their surface, but young enough not to show a large hydrogen depletion (and helium enrichment). As a consequence, we identify them as the slightly advanced versions of very massive objects with spectral types earlier than O4-6 supergiants and still in the hydrogen burning phase.

Another interesting property of these WN7-9h stars concerns their winds. As shown in Fig. 4, they follow a very well defined modified wind momentum - luminosity relation. 


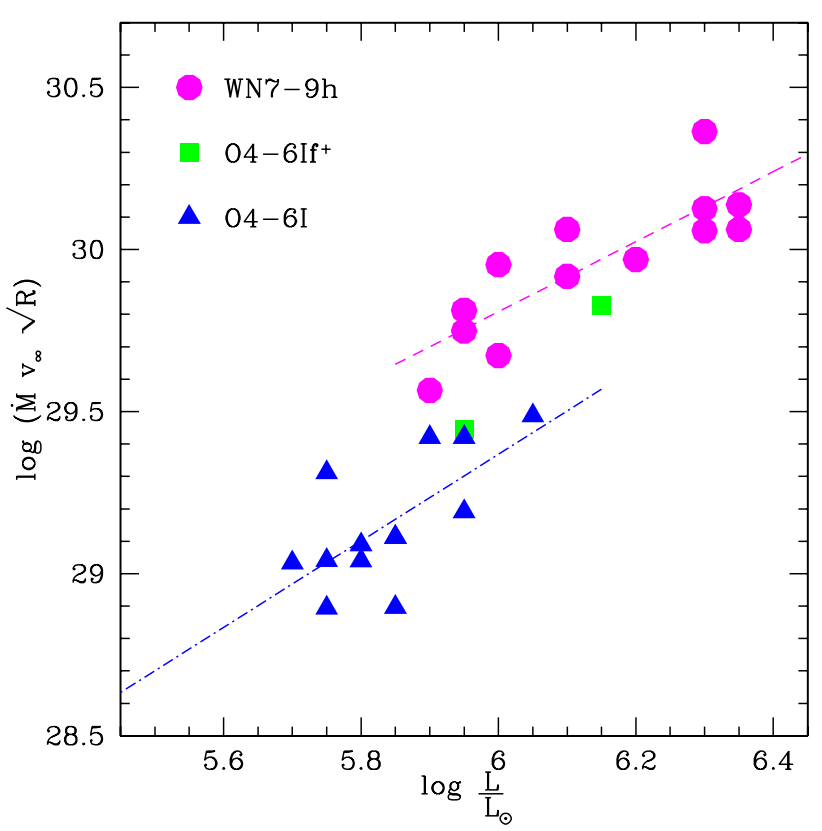

Figure 4. Modified wind momentum - Luminosity relation for the Arches stars. Blue triangles are $\mathrm{O}$ supergiants, green squares are $\mathrm{OIf}^{+}$stars, and magenta circles are WN7-9h stars. The latter stars follow a well defined relation, indicating that their winds are most likely radiatively driven.

This relation is shifted and has a different slope compared to that followed by $\mathrm{O}$ supergiants. This is however a strong indication that their winds are radiatively driven. This is another indirect evidence that these extreme objects are quite similar to $\mathrm{O}$ stars.

\section{Summary}

We have derived the stellar and wind properties of 46 stars in the central and Arches cluster in the Galactic Center. Since these clusters have different ages, they host different populations. We have thus analyzed different types of massive stars: very massive $\left(M \sim 60-120 \mathrm{M}_{\odot}\right)$ and luminous objects in the Arches cluster, older (6 Myr) objects with initial masses in the range $30-60 \mathrm{M}_{\odot}$ in the central cluster. From the detailed study of their abundances, we have been able to define evolutionary sequences between different class of objects. Fig. 5 illustrates this point. The hydrogen mass fraction of various types of objects is shown as a function of luminosity. The Geneva evolutionary tracks with rotation are overplotted (solid lines). From such a diagram, and using the predicted sequences, one can define the following sequences:

$$
\begin{array}{ll}
M \sim 30-60 \mathrm{M}_{\odot}: & \text { Ofpe } / \mathrm{WN} 9 \rightarrow \mathrm{WN} 8 \rightarrow \mathrm{WN} 8 / \mathrm{WC} 9 \rightarrow \mathrm{WC} 9 \\
M>70 \mathrm{M}_{\odot}: & <\mathrm{O} 4-6 \mathrm{I} \rightarrow \mathrm{WN} 7-9 \mathrm{~h}
\end{array}
$$

These sequences are in qualitative agreement with those summarized by Crowther (2007) in his recent review on Wolf-Rayet stars. In the particular cases studied here, we have been able to refine the sequences and to show the relation between different spectral subtypes. This illustrates the power of analysis of large sample of stars in clusters to 


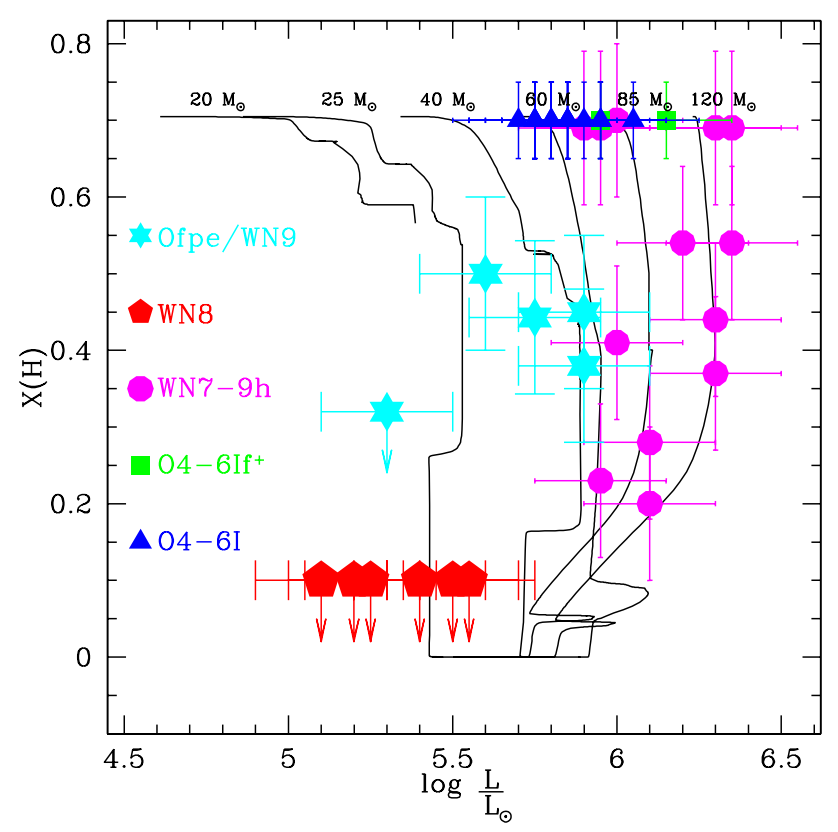

Figure 5. Hydrogen mass fraction as a function of luminosity for most of the stars studied in the Arches and central cluster. Solid lines are the recent Geneva evolutionary tracks including rotation (Meynet \& Maeder (2005)). Studying clusters of different ages allows to populate different parts of this diagram and to pinpoint the evolutionary link between various types of stars.

constrain stellar evolution. One of the most important conclusion is that in the central cluster, there is no peculiar stellar evolution as believed until recently.

\section{References}

Allen, D. A. Hyland, A. R., \& Hillier, D. J. 1990, MNRAS, 244, 706

Blum, R. D. Schaerer, D., Pasquali A. et al. 2001, AJ, 122, 1875

Crowther, P. A. 2007, ARA\&A, 45, 177

Eisenhauer, F. Genzel, R., Alexander, T. et al. 2005, ApJ, 628, 246

Figer, D. F. Najarro, F., Gilmore, D. et al. 2002, ApJ, 581, 258

Forrest, W. J., Shure, M. A., Pipher, J. L., \& Woodward, C. E. 1987, in: D. C. Backer (ed.), The Galactic Center (New York: AIP), AIP Conf Proc 155, 153

Ghez, A., Duchêne, G., Matthews, K. et al. 2003, ApJ, 586, L127

Hillier, D. J. \& Miller, D. L. 1998, ApJ, 496, 407

Martins, F., Schaerer, D., \& Hillier, D. J. 2005, A\&A A, 436, 1049

Martins, F., Genzel, R., Hillier, D. J. et al. 2007, $A \mathscr{G} A, 468,233$

Martins, F., Hillier, D. J., Paumard, T. et al. 2008, A\&A, 478, 219

Meynet, G. \& Maeder, A. 2005, A\& A, 429, 581

Najarro, F. Hillier, D. J., Kudritzki, R.-P. et al. 1994, A\&A, 285, 573

Najarro, F., Krabbe, A. Genzel, R. et al. 1997, A\&A A, 325, 700

Paumard, T. Genzel, R., Martins, F. et al. 2006, ApJ, 643, 1011 


\section{Discussion}

HAnson: You did not mention the very luminous red supergiant IRS7, in the Galactic central cluster. For the mass range and ages you list, it seems consistent with being coeval, do you agree?

Martins: Yes, I agree. The central cluster is $6 \pm 2$ Myr old. This corresponds to an age at which the first red supergiants appear. At the same time, there is still a number of Wolf-Rayet objects coming from stars with initial masses of 40-50 $\mathrm{M}_{\odot}$.

HANSON: Now that you have a deeper study of the central cluster, is the very high number of HeI emission line stars, seen initially because of observational selection effect, no longer anomalous?

MarTins: The so-called HeI emission line stars were initially discovered because they the most luminous stars in the near-IR (due to their low effective temperature). We have uncovered 10-15 times more hotter massive stars, including WN, WC and O stars. These stars are fainter in the near IR and were not seen initially. Consequently, the HeI stars are outnumbered by other types of stars and do not represent a peculiar population as thought a decade ago. Note also that the HeI stars have been observed in other environments (especially the Magellanic clouds) since their discovery in the Galactic Center.

Moffat: You indicated that the WN7-9h stars are preceded by O4-6I stars. But is this necessary? Could the WNLh stars not have started as WNLh with strong emission lines because of their high luminosity even on the ZAMS? E.g. NGC 3603 has an age of 1 Myr, barely enough to evolve an O star to WN.

Martins: Given that we see both WN7-9h and O4-6I stars in the Arches, I argued that the former probably came from stars earlier than O4-6I stars, maybe O2-3 stars. One cannot exclude that very luminous stars such as the one observed in the Arches cluster or NGC 3603 were born as WNLh stars, but the evidence I showed that WNLh stars are already evolved in terms of $\mathrm{CNO}$ processing (i.e. they are $\mathrm{N}$ rich/ $\mathrm{C}$ poor) indicate that there must exist a class of objects with almost the same luminosity, but different surface abundance patterns. Such objects could be very early O stars. Note also that the timescale to see significant $\mathrm{N}$ enrichment is of the order of $1 \mathrm{Myr}$. This could explain the presence of WNLh stars in NGC3603. 


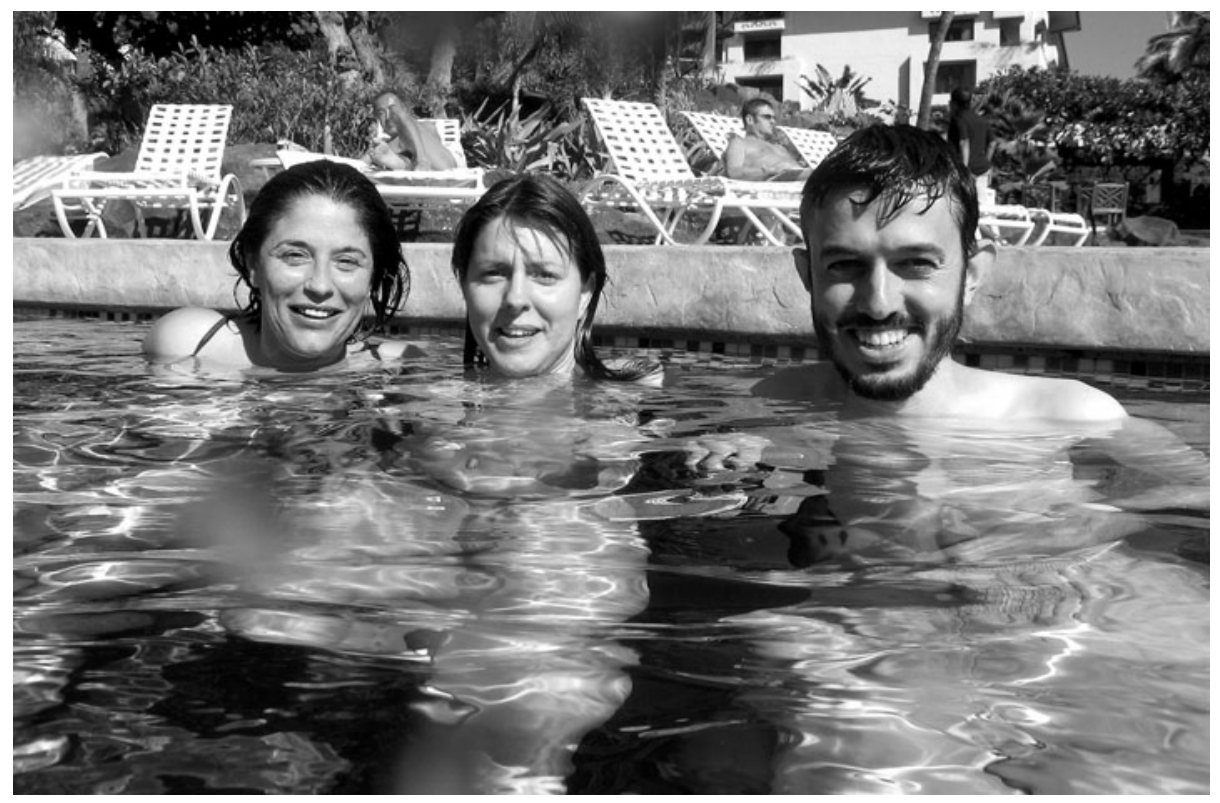

From left to right: Amparo Marco, Ana Ursúa and Alfredo Sota.

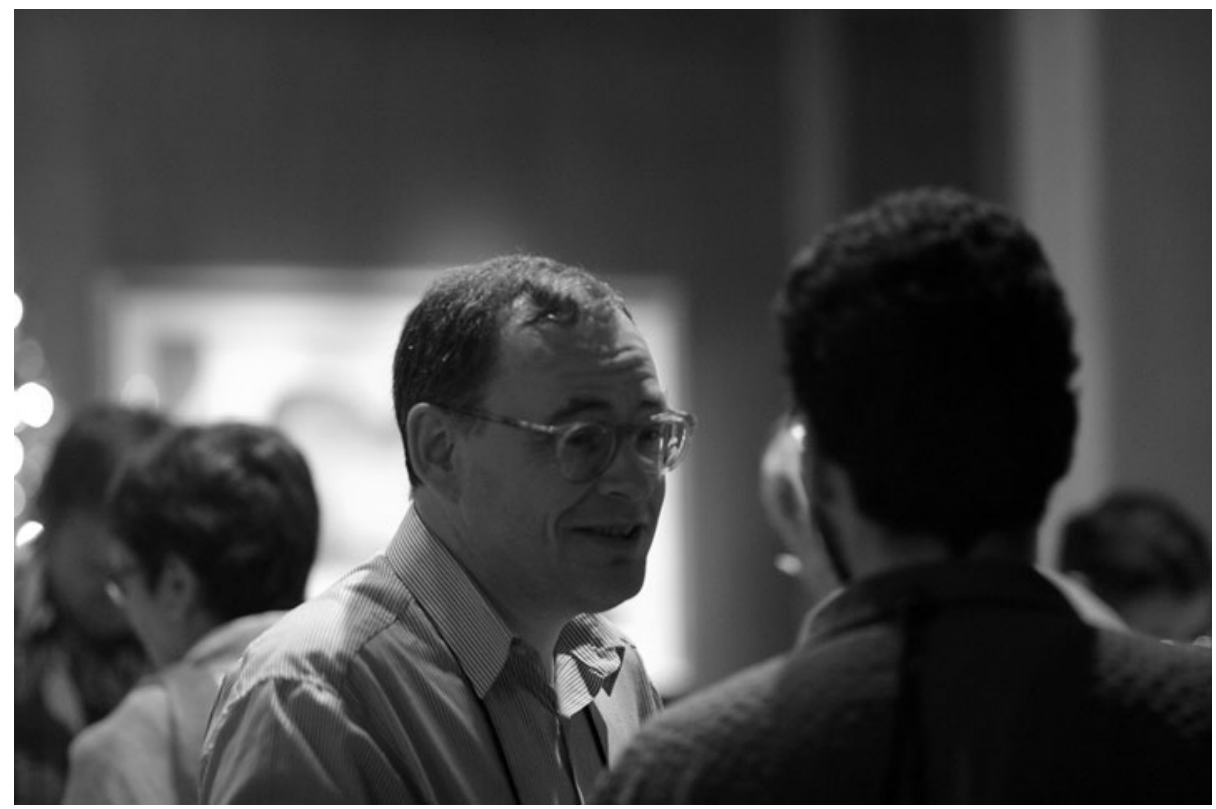

Ronny Blomme. 